\title{
The effect of boundary conditions in the numerical solution of 3-D thermoelastic problems
}

\author{
JUAN JOSÉ ANZA and ENRIQUE ALARCÓN
}

\section{INTRODUCTION}

After the extensive research on the capabilities of the Boundary Integral Equation Method produced during the past years the versatility of its applications has been well founded. Maybe the years to come will see the in-depth analysis of several conflictive points, for example, adaptive integration, solution of the system of equations, etc. This line is clear in academic research. ${ }^{1-6}$

In this paper we comment on the incidence of the manner of imposing the boundary conditions in 3-D coupled problems. Here the effects are particularly magnified: in the first place by the simple model used (constant elements) and secondly by the process of solution, i.e. first a potential problem is solved and then the results are used as data for an elasticity problem. The errors add to both processes and small disturbances, unimportant in separated problems, can produce serious errors in the final results.

The specific problem we have chosen is especially interesting. Although more general cases (i.e. transient, ${ }^{7,8}$ ) can be treated, here the domain integrals can be converted into boundary ones and the influence of the manner in which boundary conditions are applied will reflect the whole importance of the problem.

\section{THE STATIONARY THERMOELASTIC PROBLEM}

As is well known the general thermoelastic problem is represented by a set of coupled differential equations that have to be simultaneously solved in order to obtain the field of temperatures and the field of stresses and strains inside the body under study.

If it is possible to assume a stationary situation the problem can be solved in two steps; in the first one a Poisson type equation

$$
\theta_{, j i}+\frac{Q}{\lambda_{0}}=0
$$

describes the field of temperatures. Here

$$
\begin{aligned}
\theta(x, y, z) & =\text { field of temperatures } \\
Q(x, y, z) & =\text { intensity of heat emission } \\
\lambda_{0} & =\text { material conductivity constant }
\end{aligned}
$$

When $\theta$ is obtained, the Duhamel's analogy allows the solution of the problem by solving an equivalent problem (Fig. 1):

$$
\begin{aligned}
(\lambda+\mu) u_{j, j i}+\mu u_{i, j j}+X_{i}=0 & \text { in } \Omega \\
\sigma_{i j} n_{j}=t_{i} & \text { in } \partial \Omega_{1} \\
u_{i}=u_{i} & \text { in } \partial \Omega_{2} \\
\partial \Omega=\partial \Omega, U \partial \Omega_{2} U R &
\end{aligned}
$$

when $R$ is a set of zero measure.

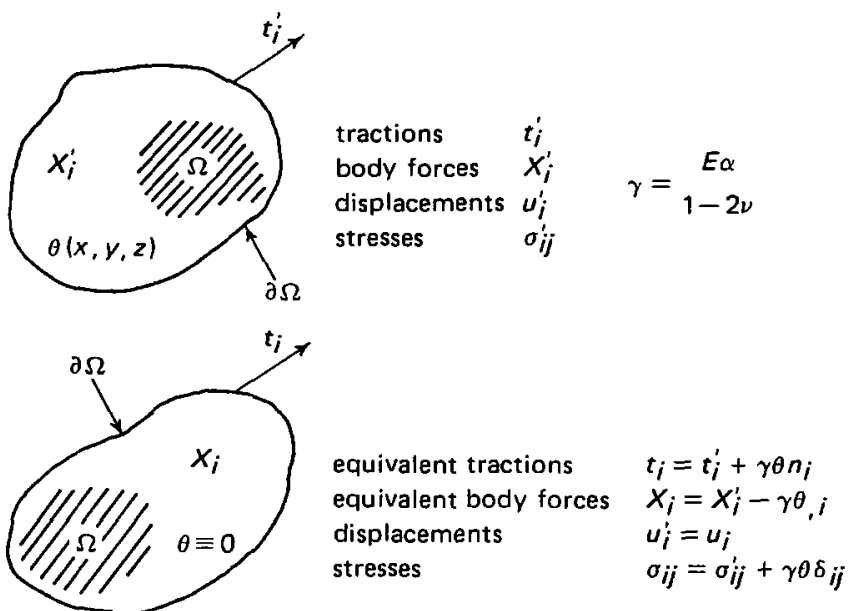

Figure 1

As is well known the representation formula for problem (1) is:

$$
\begin{aligned}
c(\mathrm{x}) \theta(\mathrm{x}) & +\int_{\partial \Omega} \frac{\partial \psi}{\partial n}(\mathrm{x}, \mathrm{y}) \theta(\mathrm{y}) \mathrm{d} s(\mathrm{y}) \\
& =\int_{\partial \Omega} \psi(\mathrm{x}, \mathrm{y}) \frac{\partial \theta}{\partial n}(\mathrm{y}) \mathrm{d} s(\mathrm{y})-\int_{\Omega} \psi(\mathrm{x}, \mathrm{y}) \nabla^{2} \theta \mathrm{d} v(\mathrm{y})
\end{aligned}
$$

$$
\psi(\mathrm{x}, \mathrm{y})=\frac{1}{4 \pi r(\mathrm{x}, \mathrm{y})}
$$

$\mathbf{n}=$ unit normal vector, $0 \leqslant c(\mathbf{x}) \leqslant 1$ while that corresponding to problem (2) is:

$$
\begin{aligned}
c_{i j}(\mathbf{x}) u_{i}(\mathrm{x}) & +\int_{\partial \Omega} T_{j i}(\mathbf{x}, \mathbf{y}) u_{i}(\mathrm{y}) \mathrm{d} s(\mathrm{y}) \\
& =\int_{\partial \Omega} U_{i i}(\mathrm{x}, \mathrm{y}) t_{i}(\mathrm{y}) \mathrm{d} s(\mathrm{y})+\int_{\Omega} X_{i} U_{i i}(\mathrm{x}, \mathrm{y}) \mathrm{d} v(\mathrm{y})
\end{aligned}
$$

$$
0 \leqslant c_{i j} \leqslant 1 \quad i, j=1,2,3
$$

and the integral kernels are defined by

$$
\begin{gathered}
U_{i i}(\mathrm{x}, \mathrm{y})=\frac{1}{16 \pi G(1-\nu) r(\mathrm{x}, \mathrm{y})}\left[(3-4 \nu) \delta_{i j}+r_{, i} r_{, j}\right] e_{j} \\
i, j=1,2,3 \\
T_{i i}(\mathrm{x}, \mathrm{y})=\frac{-1}{8 \pi(1-\nu) r^{2}(\mathrm{x}, \mathrm{y})}\left\{\frac{\partial r}{\partial n}\left[(1-2 \nu) \delta_{i j}+3 r_{, i} r_{, j}\right]\right\} \\
+(1-2 \nu)\left(n_{j} r_{, i}-n_{i} r_{, j}\right)
\end{gathered}
$$


When the volume forces are exclusively due to thermal effects it is possible to write ${ }^{9-11}$ (4) as:

$$
\begin{aligned}
& c_{i j} u_{j}(\mathrm{x})+ \int_{\partial \Omega} T_{j i}(\mathrm{x}, \mathrm{y}) u_{i}(\mathrm{y}) \mathrm{d} s(\mathrm{y}) \\
&= \int_{\partial \Omega} U_{j i}(\mathrm{x}, \mathrm{y}) t_{i}(\mathrm{y}) \mathrm{d} s(\mathrm{y})-\gamma \int_{\partial \Omega} W_{, j}(\mathrm{x}, \mathrm{y}) \\
& \times \frac{\partial \theta}{\partial n}(\mathrm{y}) \mathrm{d} s(\mathrm{y})+\gamma \int_{\partial \Omega} \theta(\mathrm{y}) W_{, j i}(\mathrm{x}, \mathrm{y}) n_{i} \mathrm{~d} s \\
&-\gamma m \int_{\partial \Omega} W(\mathrm{x}, \mathrm{y}) n_{j} \mathrm{~d} s \\
& i, j=1,2,3
\end{aligned}
$$

where

$$
\begin{aligned}
\gamma & =E \alpha / 1-2 \nu \\
\nabla^{2} \theta & =m \\
W_{i} & =(1-2 \nu)(1+\nu) r e_{i} / 8 \pi E(1-\nu)
\end{aligned}
$$

$e_{i}$ being the unit vector in the coordinate direction ' $i$ '.

\section{THE BIEM - CONSTANT ELEMENTS}

The discretisation of the previous equations as well as the geometry of the boundary domain leads to the system of equations typical of this method.,12 In our case we have chosen the simplest approach, i.e. the boundary is substituted by a series of $N$ plane triangles defined by sets of three points contained on the real surface. Moreover the evolution of tractions and displacements is assumed as constant throughout every boundary element, and the values associated to a selected point inside it, for instance its centre of gravity.

The equations corresponding to problem (1) are:

$$
\begin{gathered}
c_{l} \theta\left(\mathrm{x}_{l}\right)+\sum_{k=1}^{N} A^{l k} \theta\left(\mathbf{x}_{k}\right)=\sum_{k=1}^{N} B^{l k} \frac{\partial \theta\left(\mathbf{x}_{k}\right)}{\partial n} \\
A^{l k}=\int_{\partial \Omega_{k}} \frac{\partial \psi}{\partial n}\left(\mathrm{x}_{l}, \mathrm{y}\right) \mathrm{d} s(\mathrm{y}) \\
B^{l k}=\int_{\partial \Omega_{k}} \psi\left(\mathrm{x}_{l}, \mathrm{y}\right) \mathrm{d} s(\mathrm{y})
\end{gathered}
$$

$\mathrm{x}_{l}$ centre of gravity of element $l$

$$
l, k=1,2, \ldots, N
$$

In a problem with boundary conditions of the Dirichlet type, the coefficients of the system of equations to be solved are the $B^{l k}$ type, while conversely, for Neumann conditions, they are the $A$ type. In a Newton (or Robin) problem, there is a linear combination of both $A^{l k}$ and $B^{l k}$, while in a mixed problem there are some of the $A^{l k}$ type and others of the $B^{i k}$ type.

In a pure Neumann problem it is necessary to add one condition because the solution is undetermined. Usually this is done by fixing the value of the potential in a point and in this sense it can be said that the problem has been transformed into a mixed type one.

:Similarly the elastic problem can be formulated as follows:

$$
\begin{array}{rl}
c_{j l} u_{j}\left(\mathrm{x}_{l}\right)+\sum_{k=1}^{N} A_{j i}^{l k} u_{i}\left(\mathrm{x}_{k}\right) & \\
=\sum_{k=1}^{N} B_{j i}^{l k} t_{i}\left(\mathrm{x}_{k}\right)+\gamma \sum_{k=1}^{N}\left[D_{j i}^{l k} n_{i}^{k} \theta\left(\mathrm{x}_{k}\right)-C_{j}^{l k} \frac{\partial \theta}{\partial n}\left(\mathrm{x}_{k}\right)\right] \\
A_{j i}^{l k}=\int_{\partial \Omega_{k}} T_{j i}\left(\mathrm{x}_{l} \mathrm{y}\right) \mathrm{d} s(\mathrm{y}) \\
B_{j i}^{l k}=\int_{\partial \Omega_{k}} U_{j i}\left(\mathrm{x}_{l}, \mathrm{y}\right) \mathrm{d} s(\mathrm{y}) \\
C_{j}=\int_{\partial \Omega_{k}} W_{, j}\left(\mathrm{x}_{l}, \mathrm{y}\right) \mathrm{d} s(\mathrm{y}) \\
D_{i j}=\int_{\partial \Omega_{k}} W_{, j i}\left(\mathrm{x}_{l}, \mathrm{y}\right) \mathrm{d} s(\mathrm{y}) \\
j=1,2,3 & k, l=1,2, \ldots, N
\end{array}
$$

As with the potential case it is possible to define pure problems controlled by $A$ or $B$ coefficients, as well as linear combinations and mixed conditions. For the same reason it is not possible to solve a problem in which all boundary conditions are defined as tractions because the displacements contain rigid body displacements. To solve this difficulty some convenient displacements are fixed in order to obtain the kinematic determinacy of the body, and in this sense the problem is usually transformed into a mixed type one.

\section{EXAMPLES}

In order to analyse the results a computer program described elsewhere ${ }^{9}$ was written for a UNIVAC $1108 \mathrm{com}$ puter. In all cases material constants were chosen as:

$$
\begin{aligned}
& \nu=0.25 \\
& \mu=1 \\
& \alpha=1
\end{aligned}
$$

and problems selected so that a closed-form solution was available to compare results.

Figure 2 shows the discretisation used to model a piece of a thick-walled cylinder submitted to an interior temperature of $100^{\circ}$ and an exterior one of $20^{\circ}$. Due to the symmetry the normal displacements in the four plane faces are zero, as well as the corresponding fluxes.

In Fig. 3 we present the evolution of the temperature along the radius in solid line while points are the computed values. It is seen that the latter are very accurate in comparison with the closed-form solution. In the same way Fig. 4 presents the computed results of the radial displacement. Here the small errors in the input temperature manifest themselves in larger errors especially near the exterior boundary where the discretisation is rougher. Nevertheless the relative differences are not very large as can be seen in the numerical results of Table 1 . The same effect is apparent in Fig. 5 and Table 2 which illustrate the evolution of radial, hoop and axial stresses versus the radial coordinate. While hoop and axial stresses are relatively well approximated, radial stresses present some deviation from the theoretical results reaching important relative errors. If as is usually said B.I.E.M. is advantageous from the point 


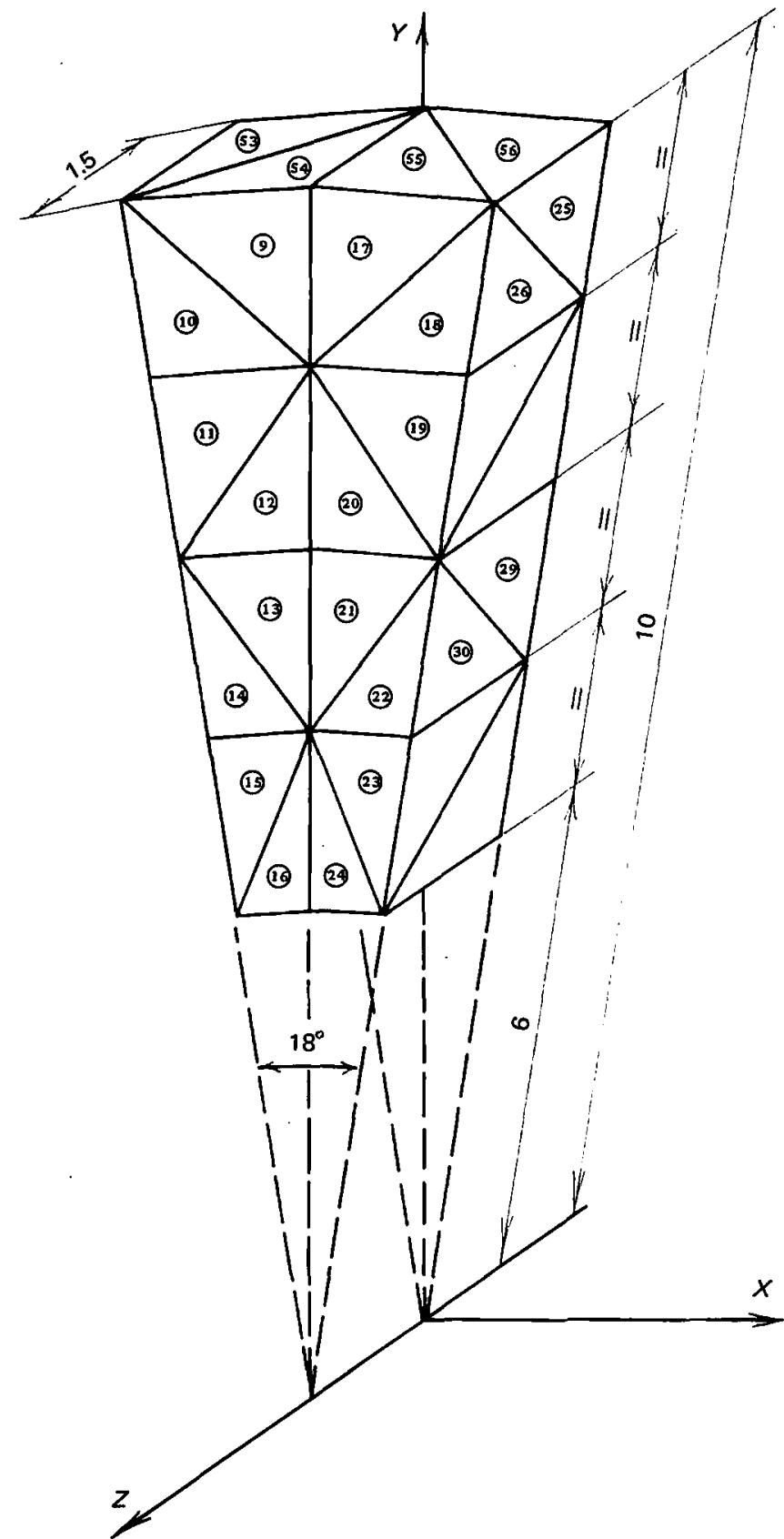

Figure 2

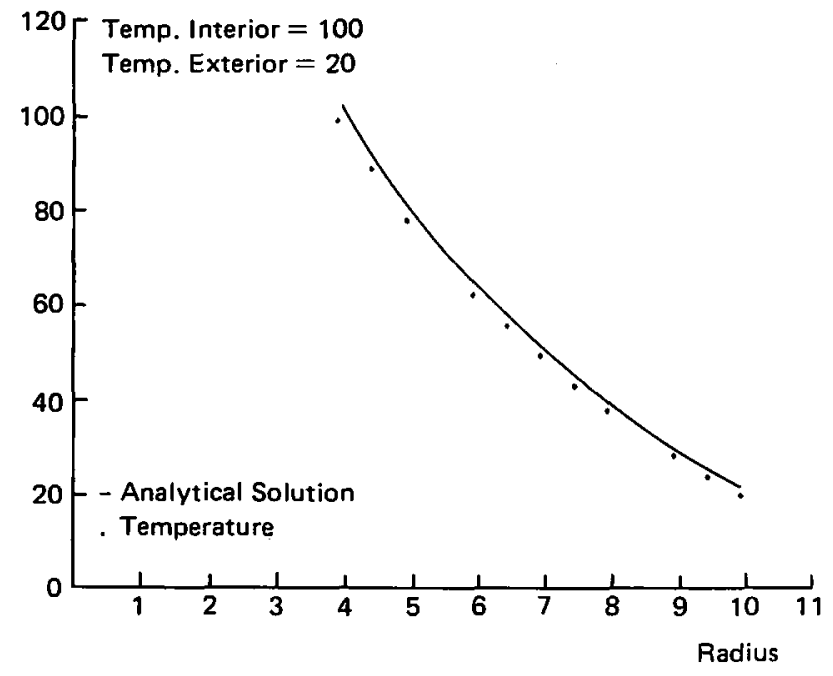

Figure 3

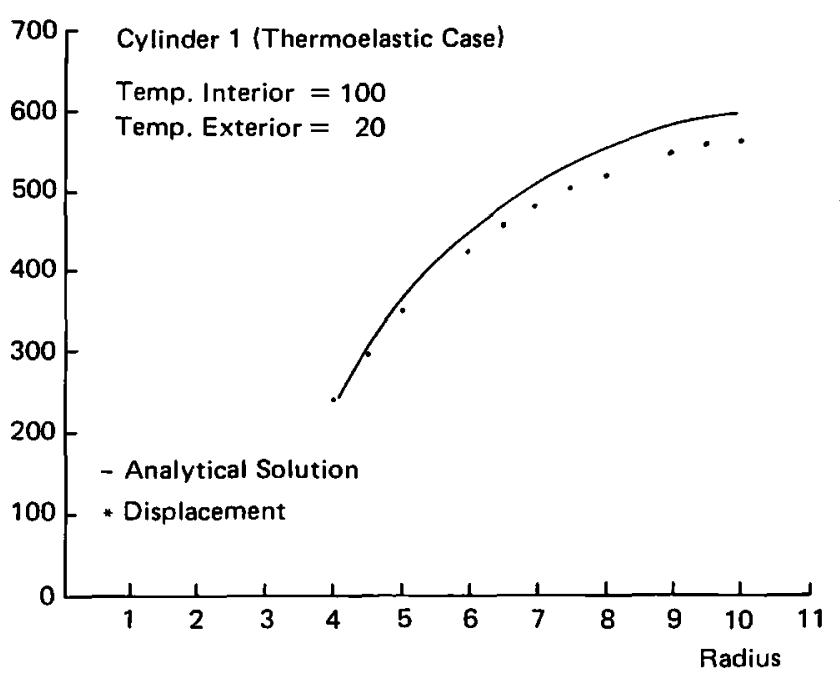

Figure 4

Table I

\begin{tabular}{ccccccc}
\hline & \multicolumn{2}{c}{ Radial displacement } & & \multicolumn{2}{c}{ Radial displacement } \\
\cline { 3 - 3 } \cline { 5 - 6 } Radjus & Theoretical & Comp. & Radius & Theoretical & Comp. \\
\hline 4 & 242.07 & 242.61 & 7.5 & 536.80 & 503.08 \\
4.5 & 308.72 & 298.20 & 8 & 556.90 & 521.82 \\
5 & 364.27 & 349.98 & 9 & 586.86 & 549.07 \\
6 & 450.66 & 425.13 & 9.5 & 597.36 & 558.93 \\
6.5 & 484.28 & 457.26 & 10 & 605.20 & 561.93 \\
7 & 412.77 & 482.46 & & & \\
\hline
\end{tabular}

Cylinder 1 (Thermoelastic Case)

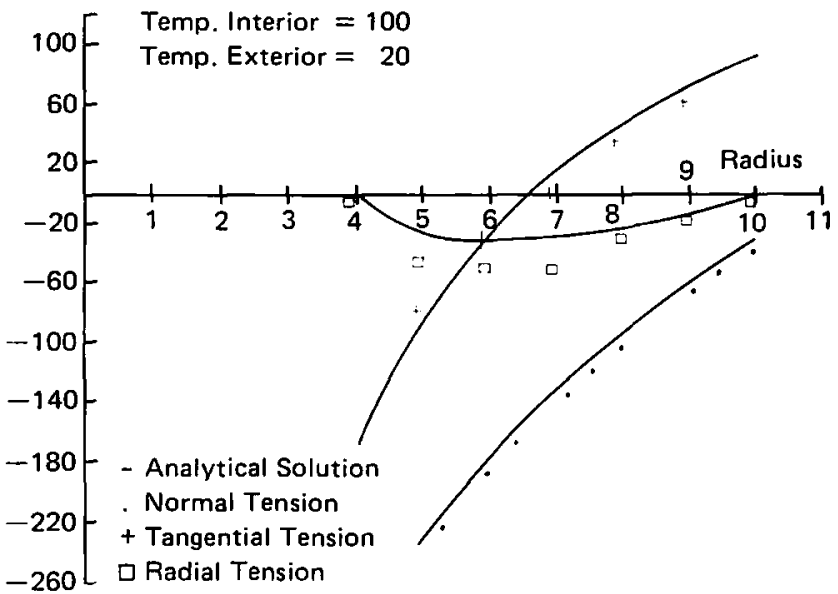

Figure'5

Table 2

\begin{tabular}{|c|c|c|c|c|c|c|}
\hline \multirow[b]{2}{*}{ Radius } & \multicolumn{2}{|c|}{ Radial stress } & \multicolumn{2}{|c|}{ Hoop stress } & \multicolumn{2}{|c|}{ Axial stress } \\
\hline & Theor. & Comp. & Theor. & Comp. & Theor. & Comp. \\
\hline 4.5 & & & -121 & -10 & & \\
\hline 5 & -24.67 & -42.11 & -82. & -74.46 & -228.05 & -206.70 \\
\hline 6 & -29 . & & -24.7 & -23.41 & -174.98 & -161.93 \\
\hline 6.5 & & & -2.68 & -0.01 & -151.69 & -132.97 \\
\hline 7 & -25.47 & -45.50 & 16.39 & 14.74 & -130.12 & -120.42 \\
\hline 7.5 & & & 33.11 & 33.90 & -110.04 & -100.55 \\
\hline 8 & -18.18 & -24.94 & 47.96 & 39.44 & -91.26 & -76.78 \\
\hline 9 & -9.37 & -15.94 & 73.43 & 66.90 & -56.98 & -47.02 \\
\hline 9.5 & & & 84.51 & 87.64 & -41.25 & -31.8 \\
\hline
\end{tabular}




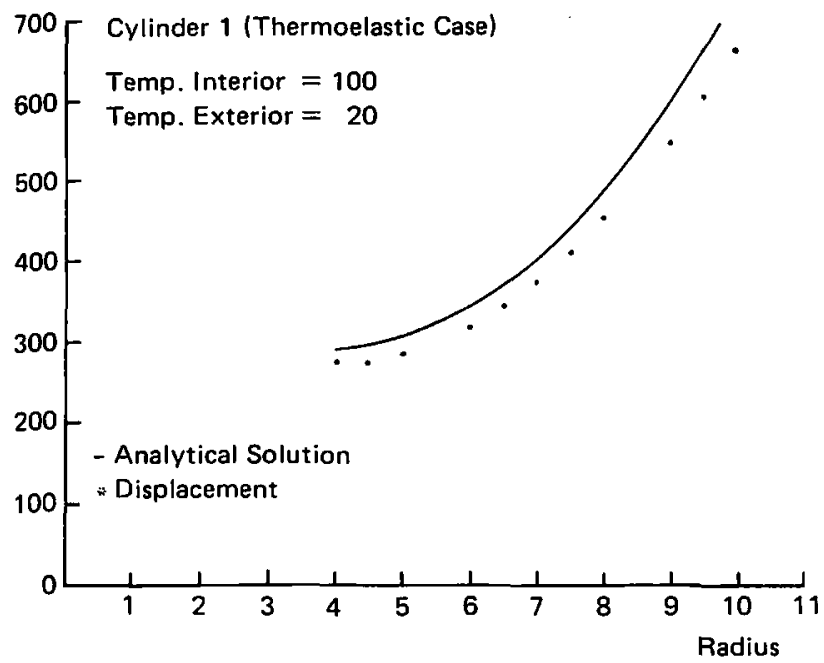

Figure 6

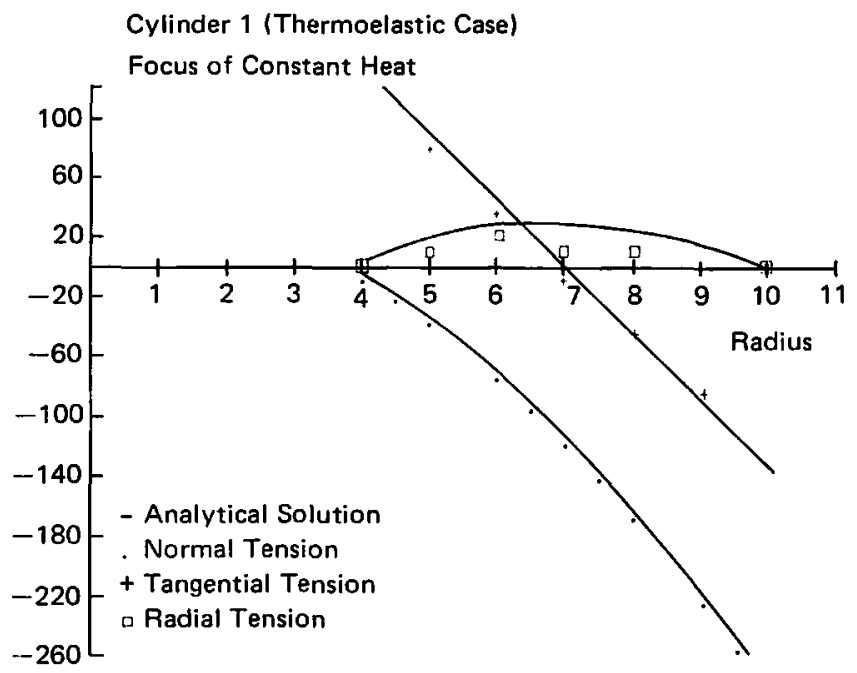

Figure 7

Table 3

\begin{tabular}{ccccccc}
\hline & \multicolumn{2}{c}{ Radial displacement } & & \multicolumn{2}{c}{ Radial displacement } \\
\cline { 2 - 3 } \cline { 5 - 6 } Radius & Theoretical & Comp. & Radius & Theoretical & Comp. \\
\hline 4 & 290.23 & 276 & 7.5 & 447.35 & 412 \\
4.5 & 295.19 & 275 & 8 & 491.73 & 454 \\
5 & 306.68 & 286 & 9 & 597.78 & 547 \\
6 & 346.85 & 320 & 9.5 & 659.89 & 604 \\
6.5 & 375.01 & 347 & 10 & 728.37 & 664 \\
7 & 408.49 & 376 & & & \\
\hline
\end{tabular}

Table 4

\begin{tabular}{|c|c|c|c|c|c|c|}
\hline \multirow[b]{2}{*}{ Radius } & \multicolumn{2}{|c|}{ Radial stress } & \multicolumn{2}{|c|}{ Hoop stress } & \multicolumn{2}{|c|}{ Axial stress } \\
\hline & Theor. & Comp. & Theor. & Comp. & Theor. & Comp. \\
\hline 4.5 & & & 111.81 & 103 & -19.1 & -19.4 \\
\hline 5 & $22^{\prime} 5$ & 7.65 & 87.47 & 79 & -34.92 & -29 \\
\hline 6 & $29^{\prime} 63$ & 20.25 & 43.79 & 36 & -71.55 & -64 \\
\hline 6.5 & & & 22.75 & 21 & 21 & -81 \\
\hline 7 & $28^{\prime} 62$ & 1.72 & 1.59 & -5.76 & -114.84 & -103 \\
\hline 7.5 & & & -19.97 & -19 & -138.98 & -127 \\
\hline 8 & $22 ' 5$ & 9.13 & -42.14 & -43 & -164.79 & -147 \\
\hline 9 & $12^{\prime} 71$ & 10.63 & -88.84 & -84.5 & -221.40 & -199 \\
\hline 9.5 & & & -113.56 & -90 & -252.20 & -216 \\
\hline
\end{tabular}

of view of accuracy in the determination of stresses it is worth examining this problem which manifests itself even in such a simple example.

As was suggested above the symmetric conditions here are imposed by annealing rows and columns associated with the corresponding degrees of freedom, and this process can affect the conditioning of the matrix.

In Figs. 6 and 7 and Tables 3 and 4 the same effects are observed.

Here the problem is a bit different and corresponds to the case in which the cylinder is full of source heat points uniformly distributed. The temperature has the form:

$$
\theta=k r^{2} \quad k=1
$$

and the governing equation is:

$$
\nabla^{2} \theta=4 k
$$

Taking $k=1$ it is seen that although the temperature is well approximated, the displacements (Fig. 6) present some deviation which is accentuated again in the radial stresses (Fig. 7 and Table 4).

To observe the influence that the imposition of boundary conditions has in the matrix, the very simple example of Fig. 8 has been solved for different temperature distributions. It is assumed that the hexahedron is fixed in the unseen faces by spheres constraining the normal displacements, and this conditioning destroys the general symmetry of the matrix and introduces a new one with respect normal to the main diagonal. Even assuming a constant temperature of say $100^{\circ}$ the obtained displacements are in error and this effect is more pronounced when the temperature is assumed to follow the linear law

$$
\theta=40 x
$$

The displacements are then (stresses are zero everywhere):

$$
\begin{aligned}
u & =40 \frac{x^{2}}{2} \\
v & =40 x y \\
w & =40 x z
\end{aligned}
$$

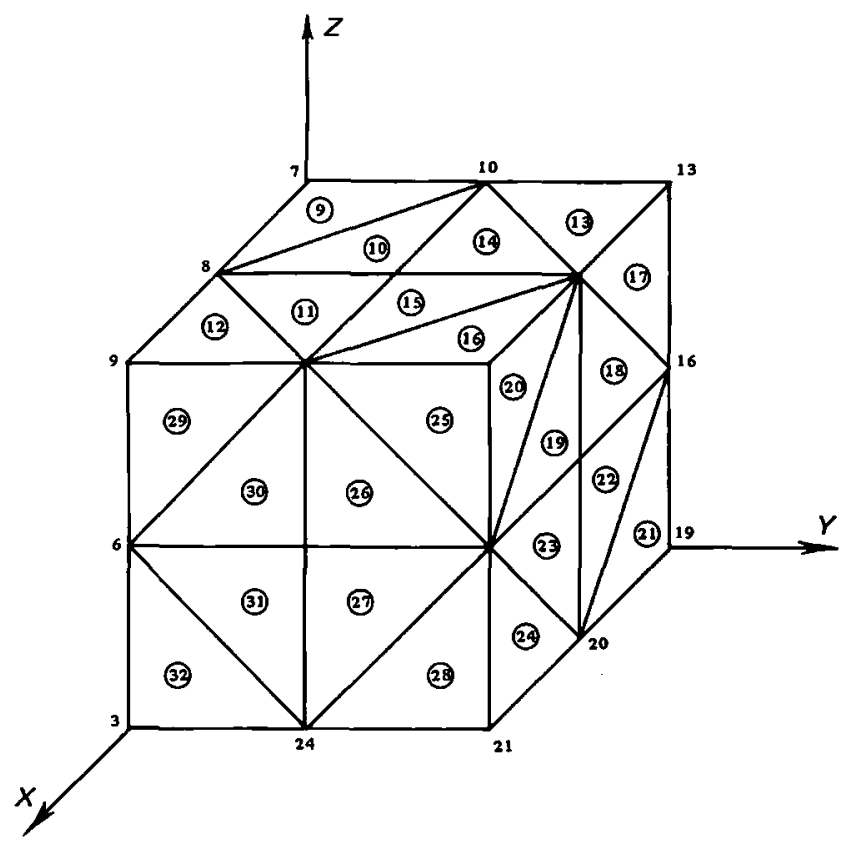

Figure 8 


\begin{tabular}{|c|c|c|c|c|c|c|}
\hline \multirow{2}{*}{$\begin{array}{l}\text { Element } \\
\text { number }\end{array}$} & \multicolumn{2}{|c|}{ Displacement $u$} & \multicolumn{2}{|c|}{ Displacement $v$} & \multicolumn{2}{|c|}{ Displacement $w$} \\
\hline & Theor. & Comp. & Theor. & Comp. & Theor. & Comp \\
\hline 9 & 5 & -15 & 10 & 13 & 60 & 90 \\
\hline 10 & 20 & -16 & 40 & 37 & 120 & 124 \\
\hline 11 & 80 & 41 & 80 & 63 & 240 & 199 \\
\hline 12 & 125 & 93 & 50 & 35 & 300 & 232 \\
\hline 33 & 180 & 158 & 60 & 30 & 300 & 203 \\
\hline 34 & 180 & 174 & 120 & 68 & 240 & 158 \\
\hline 35 & 180 & 158 & 240 & 158 & 240 & 158 \\
\hline 36 & 180 & 126 & 300 & 200 & 300 & 200 \\
\hline 37 & 180 & 193 & 60 & 32 & 60 & 32 \\
\hline 38 & 180 & 190 & 120 & 71 & 120 & 71 \\
\hline 39 & 180 & 174 & 240 & 157 & 120 & 68 \\
\hline 40 & 180 & 158 & 300 & 202 & 60 & 30 \\
\hline
\end{tabular}

Table 6

\begin{tabular}{|c|c|c|c|c|c|c|}
\hline \multirow{2}{*}{$\begin{array}{l}\text { Element } \\
\text { number }\end{array}$} & \multicolumn{2}{|c|}{ Displacement $u$} & \multicolumn{2}{|c|}{ Displacement $v$} & \multicolumn{2}{|c|}{ Stress 3} \\
\hline & Theor. & Comp. & Theor. & Comp. & Theor. & Comp. \\
\hline \multirow{5}{*}{$\begin{array}{r}9 \\
10 \\
11 \\
12\end{array}$} & -41.66 & -37.51 & nil & -1.23 & 166.66 & 180 \\
\hline & -66.66 & -62.51 & nil & -1.03 & 233.33 & 242.42 \\
\hline & -66.66 & -62.52 & nil & 1.04 & 366.66 & 357.57 \\
\hline & -41.66 & -37.51 & nil & 1.24 & 433.33 & 420 \\
\hline & \multicolumn{2}{|c|}{ Displacement $v$} & \multicolumn{2}{|c|}{ Displacement $w$} & \multicolumn{2}{|c|}{ Stress 1} \\
\hline 33 & nil & -3.39 & nil & 3.39 & -300 & -298.88 \\
\hline 34 & nil & -2.07 & nil & 2.07 & -300 & -304.68 \\
\hline 35 & nil & 2.07 & nil & 2.07 & -300 & -304.68 \\
\hline 36 & nil & 3.39 & nil & 3.39 & -300 & -296.88 \\
\hline 37 & nil & -3.39 & nil & -3.39 & -300 & -296.88 \\
\hline 38 & nil & -2.07 & nil & -2.07 & -300 & -304.68 \\
\hline 39 & nil & 2.07 & nil & -2.07 & -300 & -304.68 \\
\hline 40 & nil & 3.39 & nil & 3.39 & -300 & -296.88 \\
\hline
\end{tabular}

But the computed results are in error as shown in Table 5 .

In order to show that the kernel of the error lies in the induced asymmetry the latter example was run assuming that every face is constrained by spheres, that is, that the normal displacements are zero in the six faces. The theoretical stresses are now:

$$
\begin{aligned}
& \sigma_{x}=-300 \\
& \sigma_{y}=\sigma_{z}=-\left(\frac{400}{3} x+100\right) \alpha
\end{aligned}
$$

while the displacements are:

$$
\begin{aligned}
& u=\left(\frac{100}{3} x^{2}-100 x\right) \alpha \\
& v=w=0
\end{aligned}
$$

Table 6 shows how far the results are better now, although some errors are still present.

The problems seen previously can be eliminated auto. matically when there is symmetry in the geometry and symmetric or antisymmetric load conditions. ${ }^{13,9}$ Assuming, for instance, the hexahedron case, where there is a spherical symmetry of the displacement distribution, the values of movement as well as of its derivatives are fixed in one of its octants. In the system of equations the number of unknowns are reduced by grouping the integration constants $A_{i j}$ and $B_{i j}$ in every common variable $u_{i}$ or $t_{i}$ corresponding to one specific element and the other seven symmetric ones. In this way the problem can be solved by discretising only the three exterior faces of an octant; and the same conclusions can be drawn for the antisymmetric case.

Of course the idea is also useful in potential problems for antisymmetric cases.

To see the effectiveness of the procedure the hexahed ron example was solved again with the reduced discretisation of Fig. 9 (hidden faces are not necessary now). The variation of the temperature is assumed constant with value $100^{\circ} \mathrm{C}$.

In Table 7 selected values are presented showing the improvement obtained over the previous results.

Another curious phenomenon has been observed when treating convective (Robin-Newton) conditions. The sample problem was done on the cylinder of Fig. 2 with the following set of data:

$$
\begin{aligned}
\theta_{\text {ext }} & =20^{\circ} \mathrm{C} \text { (ambient) } \\
\theta_{\text {int }} & =100^{\circ} \mathrm{C} \text { (ambient) } \\
k & =\lambda_{0}=31 \mathrm{kcal} / \mathrm{h} . \mathrm{m}{ }^{\circ} \mathrm{C}
\end{aligned}
$$

Several cases were run for a convective problem with several combinations of the film coefficient. Maintaining

\begin{tabular}{|c|c|c|c|c|c|c|c|c|c|}
\hline \multirow{3}{*}{$\begin{array}{c}\text { Ele- } \\
\text { ment } \\
\text { No. }\end{array}$} & \multicolumn{3}{|c|}{ Displacement $\|$} & \multicolumn{3}{|c|}{ Displacement $v$} & \multicolumn{3}{|c|}{ Displacement $w$} \\
\hline & $\mathrm{Th}$ & & $o m p$ & & $\operatorname{lom}_{1}$ & & & $\mathrm{Co}$ & \\
\hline & & & $\mathrm{P}$ & & $S$ & $P$ & & & $\mathbf{P}$ \\
\hline 1 & & & & & & & & & \\
\hline 2 & 10 & & & & & & & & \\
\hline 3 & & & & & & & & & \\
\hline 4 & 25 & & & 5 & & & & & \\
\hline 5 & 50 & & & 250 & 244.1 & 231 & 30 & 285 & 27 \\
\hline 6 & 100 & 98 & & 20 & 198 & & 30 & 286 & 275 \\
\hline 7 & & & & & & & & & \\
\hline 8 & & & & 250 & 240.6 & 231.4 & 300 & 282 & 273 \\
\hline
\end{tabular}
the interior temperature at $100^{\circ} \mathrm{C}$ and the exterior one at $20^{\circ} \mathrm{C}$, those combinations were:

$$
\text { (1) } h_{\text {int }}=h_{\text {ext }}=1.200 \mathrm{kcal} / \mathrm{h} \cdot \mathrm{m}^{2}{ }^{\circ} \mathrm{C}
$$

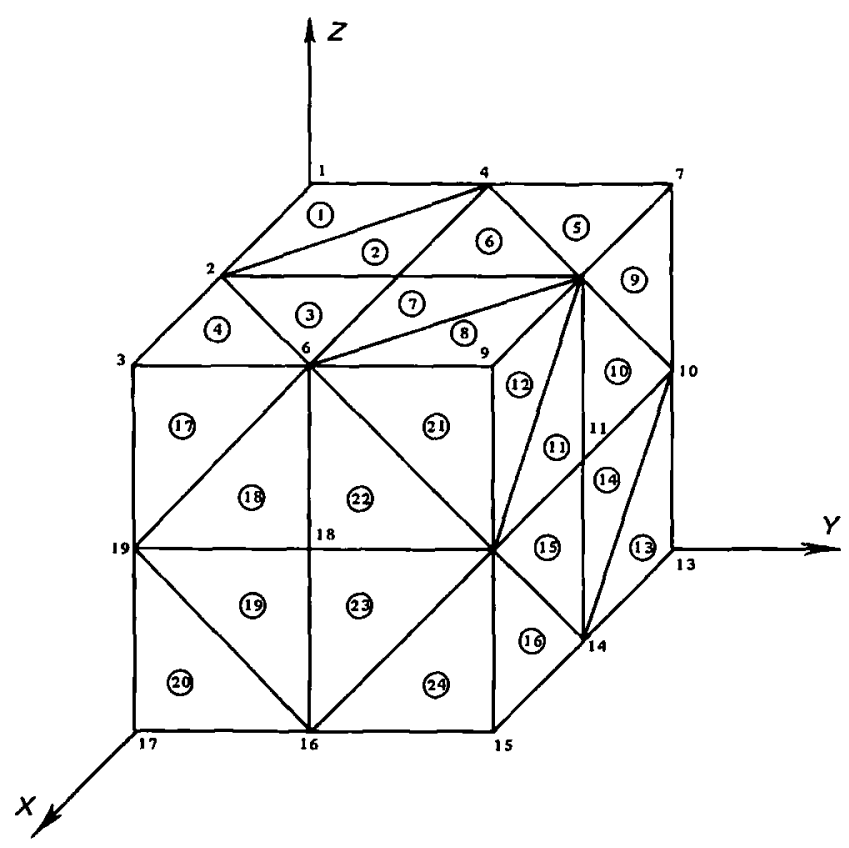

Figure 9,

Table 7 


$$
\text { (2) } \begin{aligned}
h_{\text {int }} & =8 \mathrm{kcal} / \mathrm{h} \cdot \mathrm{m}^{2}{ }^{\circ} \mathrm{C} \\
h_{\text {ext }} & =1.200 \mathrm{kcal} / \mathrm{h} \cdot \mathrm{m}^{2}{ }^{\circ} \mathrm{C} \\
\text { (3) } h_{\text {int }} & =8 \mathrm{kcal} / \mathrm{h} \cdot \mathrm{m}^{2}{ }^{\circ} \mathrm{C} \\
h_{\text {ext }} & =8 \mathrm{kcal} / \mathrm{h} \cdot \mathrm{m}^{2}{ }^{\circ} \mathrm{C}
\end{aligned}
$$

The first one produced a reasonable agreement between the theoretical and computed values, the second produced errors of the order of $5 \%$ at the interior face while the rest was correct and the third produced disparate negative radial temperatures.

As the coefficient $h / k$ is in this case very low

$$
\frac{h}{k}=\frac{0.0008}{0.31}=0.0026
$$

the first idea was to scale the final matrix in order to elimi. nate the possible bad conditioning. We tried:

(1) The classical change

$$
x=D \xi \quad D_{i i}=\frac{1}{k_{i i}} \quad D_{i j}=0
$$

or

$$
\left(D^{T} K D\right) \xi=D^{T} F
$$

(2) The scaling of the columns corresponding to the low $h$ values in order to produce values of the same order in all of them.

(3) The change of variables from absolute to relative (differences between opposite faces) values, as is usually done with nearly rigid finite elements.

(4) The solution of the system by the MCG method.

In all cases we got small differences but very bad results, showing that the system was correctly solved. Then we decided to explore the possibility of a quasi-Neumann problem; the reason is that, as $h$ is very low, the coefficient which affects the $B$ values is smaller and then the influence of $B$ is nil in comparison with the $A$ values. Then we decided to fix the value of the correct temperature at a point obtaining immediately reasonable results (Fig. 10 (3)).

The negative values modified by a constant were of the same order of precision. But it can be seen (Fig. 10(2)) that the general trend of the temperature evolution along the radius is inverted with respect to the correct one, which makes it difficult to suggest a correction for a general situation.

We decided also to explore the refinement of the coefficients $A$ and $B$ by a more careful computation by subdividing the elements. In this case we again obtained bad results, but oscillating around a nearly constant value (Fig. $10(4)$ ); that is, the nature of the problem is clearly seen again. faces

Finally, we tested the equilibrium condition along the

$$
\int q \mathrm{~d} a=0
$$

obtaining large errors.

In conclusion, when solving a Newton (Robin) problem it is necessary to test the condition $\int q \mathrm{~d} a$ before the results are confirmed. A close inspection of the results can show the reference potential level, but in general, it would be better to incorporate (15) as an additional equation in the manner indicated, for instance, by Symm ${ }^{14}$ (Fig. 10(1)).

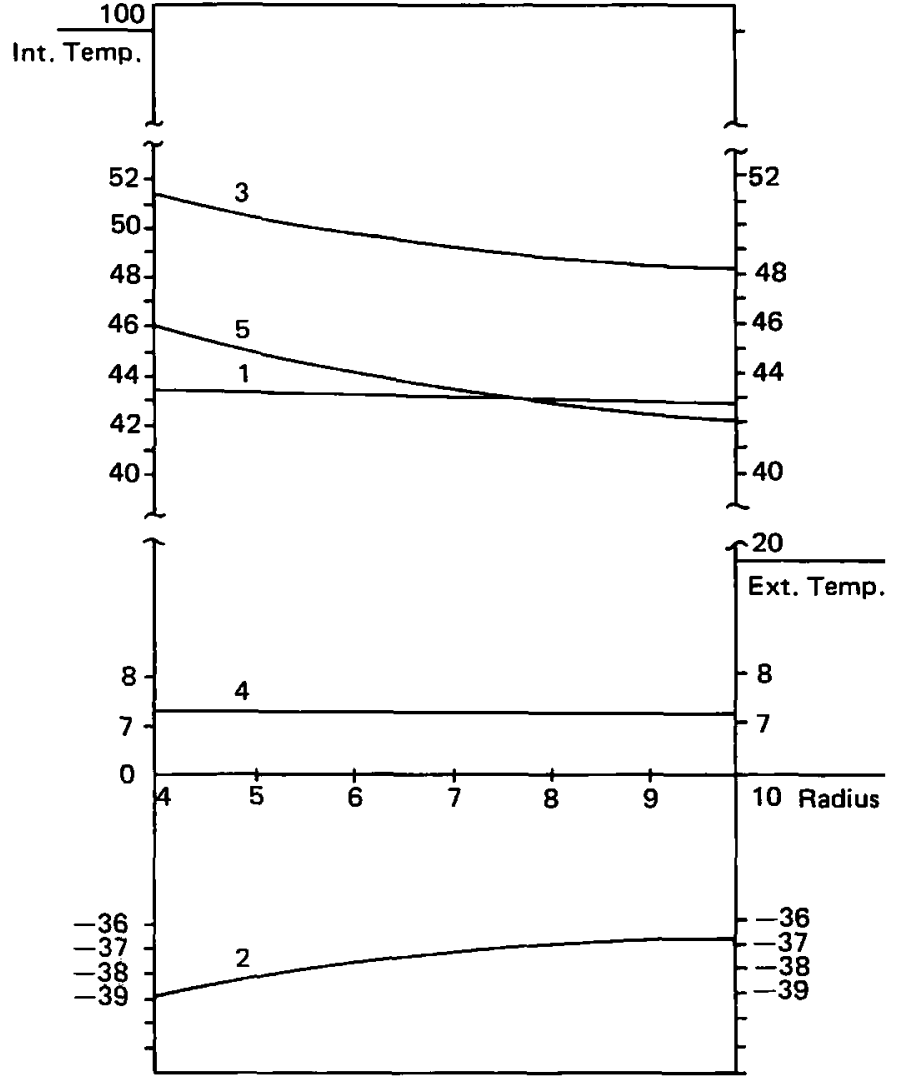

(1) Theoretical distribution and linear least squares solution.

(2) Direction solution without any precaution.

(3) Solution after fixing temperature at point 56.

(4) Direct solution after constants refinement.

(5) Solution by direct establishment of flux condition.

Figure 10. Temperature evolution along the radius

\section{CONCLUSIONS}

The accurate imposition of boundary conditions is essential if reliable results are to be obtained with BIEM. In this sense constant three-dimensional elements are especially sensitive to 'flexural' type actions, quasi-Neumann problems and symmetry conditions.

The recommended procedure of fixing points ${ }^{12}$ to simulate symmetry can induce bad results and then it seems better to use the automatic technique described elsewhere ${ }^{13}$ to incorporate those conditions.

In some Robin problems the boundary conditions may induce quasi-Neumann problems. The recourse to fixing a point is then inapplicable because the true temperature is unknown. The solution is to establish an 'equilibrium' condition as proposed by $\mathrm{Symm}^{14}$ although this means a special subroutine (Linear Least Squares method) to solve the problem. As Fig. 10(5)) shows, roughly accurate results can be obtained by substituting one equation by the 'equilibrium' conditions without any additional work.

Finally, the inaccuracies detected long ago ${ }^{15}$ when 3-D BIEM with constant elements is applied to 'flexural' problems, can be solved in the same way by enforcing the equilibrium conditions as supplementary equations as described above for the Robin problem.

\section{References}

1 Doblare, M. Three dimensional formulation of the Boundary Element Method with parabolic interpolation. (In Spanish.) Thesis E.T.S.I.I., Madrid, 1981 
2 Benitez, F. Formulation of boundary in tegral-equations method in three-dimensional elastoplasticity. (In Spanish.) Thesis E.T.S.I.I., Madrid, 1981

3 Martin, A. et al. Mixed elements in the boundary theory, 2nd Seminar on Recent Advances in Boundary Elements Method, Southampton, C.M.L. Publications, 1980

4 Brebbia, C. Boundary Elements Method for Engineers, Pentech Press, London, 1978

5 Pilkey, W. and Shaw, R. et al. Innovative Numerical Analysis for the Engineering Sciences, Univ. Press of Virginia, 1980

6 Brebbia, C. Boundary elements methods. Proc. of the 3rd Int. Sem., Irvine, California, Springer Verlag, 1981

7 Wrobel, L. C. and Brebbia, C. A. Axisymmetric-potential problems. Proc. of the 2nd Int. Sem. on Recent Advances in Boundary Element Methods, Southampton, March 1980

8 Roures, V. Boundary element method in transient heat transfer. To be published in Computers and Structures.

9 Anza, J. The Boundary Element Method in the theory of thermoelasticity. (In Spanish.) Thesis, E.T.S.I.I., Madrid, 1981
10 Danson, D. J. A boundary element formulation of problems in linear isotropic elasticity with body forces, 3rd Int. Sem. for Boundary Element Method, Irvine, Springer Verlag, 1981

11 Rizzo, F. and Shippy, D. The Boundary Element Method in Thermoelasticity. Developments in Boundary Elenent Methods. Ed. by Banerjee, P. K. and Butterfield, R., Applied Science Publishers, 1979

12 Alarcón, Martin, A. and Parts, F. Boundary elements in potential and elasticity theory, Computers and Structures, 1979, 10, 351, Pergamon Press

13 Lachat, J. C. and Watson, J. O. Effective numerical treatment of boundary integral equations: a formulation for threedimensional elastostatics, Int. J. for Num. Methods in Engineering, 1976, 10,991

14 Symm, G. T. The Robin problem for Laplace equation, 2nd Int. Sem. on Recent Advances in Boundary Element Methods, Southampton, C.M.L. Publications, 1980

15 Cruse, T. A. Numerical solutions in three-dimensional elastostatics, Int. J. Solids Structures, 1969, 5, 1259, Pergamon Press 